\title{
Theory of the magnetization and exchange-enhanced susceptibility of alloys. II. Zero-temperature magnetization and susceptibility in the presence of moments
}

\author{
Nicholas Kioussis* and J. W. Garland \\ Department of Physics, University of Illinois at Chicago, Chicago, Illinois 60680
}

(Received 8 December 1986)

\begin{abstract}
A theoretical treatment of the zero-temperature exchange-enhanced susceptibility of paramagnetic substitutionally disordered alloys within the random-phase approximation was presented in the preceding paper ( $I$ of this series). In the present paper that treatment is extended so as to allow the calculation of the local susceptibility in the presence of moments and/or large applied magnetic fields and the calculation of the spontaneous and induced local magnetization. The cluster treatment presented here is the first cluster theory to treat quantitatively the effect of moment formation on the local susceptibility. Moreover, the techniques presented here are computationally feasible even for the study of concentrated alloys and yield results for the size of local moments as a function of their local environment. Interpolation schemes which allow one to calculate easily the magnetization and local susceptibility associated with any magnetic cluster configuration also are presented. The use of the formalism presented is illustrated by applying it to the calculation of the magnetization and susceptibility of different configurations of $\mathrm{Ni}$ atoms embedded in $P d$ and in exchange-enhanced effective media.
\end{abstract}

\section{INTRODUCTION}

The theory presented in paper I of this series ${ }^{1}$ (hereafter referred to as I), like most previous theories ${ }^{2-5}$ for the magnetic susceptibility of alloys, applies only to the paramagnetic regime and only to linear response. In particular, all alloy generalizations of the theory of local exchange enhancement ${ }^{6}$ predict only the formation of moments of unspecified size on every site when the local susceptibility diverges within the random-phase approximation (RPA) on any site. They fail to predict either the size of the moments or the local susceptibility in the presence of those moments. Furthermore, the present theories all fail to treat the dependence of the susceptibility on the applied magnetic field.

Here, we generalize the theory presented in I, obtaining the first theory of local exchange enhancement valid on either side of the point at which the local exchange enhancement reaches criticality within the RPA. The resultant nonlinear, self-consistent cluster theory yields the spontaneous and induced local magnetization on any site as a function of the local chemical and magnetic environment of that site. Similarly, it yields a local susceptibility $\chi_{i j}(\omega)$ within the RPA which depends on the chemical occupation of and the local moments on the sites $i$ and $j$ and all other sites $k$ in the vicinity of site $i$ or site $j$. Although the theory presented here, like all other RPA theories, is not valid in the immediate vicinity of the point at which the local exchange enhancement achieves criticality, the theory is generalized further in the following paper so as to include the effects of quantum and thermal spin fluctuations in a way consistent with the results of renormalization-group theory. ${ }^{7}$ That generalization makes the theory presented in I and here valid for all values of the local exchange enhancement and for all temperatures.

The theory presented here is specifically intended for application to nondilute metallic alloys which contain local moments displaying significant local-environment effects. It is the first quantitative theory for the susceptibility and magnetization of such alloys. Many such alloy systems exist, of which the best known are perhaps $\mathrm{Ni}-\mathrm{Cu}$ and Pd-Ni. The theory has been applied to Pd-Ni by the authors ${ }^{8}$ and found to yield results in good agreement with experiment. For alloys not containing local moments, the theory presented in I is directly applicable, and the generalizations presented in this paper are unnecessary. For dilute alloys, the magnetization is given more accurately by single-site self-consistent spinpolarized alloy band calculations. ${ }^{9,10}$ Such calculations also are appropriate for those concentrated magnetic alloys which display only minor local-environment effects. ${ }^{11}$ On the other hand, cluster self-consistent spin-polarized alloy band calculations to determine moments as a function of local environment are not feasible even on the fastest computers available today. Thus, for the applications intended the theory presented here is uniquely useful.

This paper is organized as follows. In Sec. II it is shown that even in the presence of local moments there exists an integral equation for the alloy susceptibility of the same form as the basic equation of I. This result, which is given for the first time here, allows one to use the formalism of I to determine the local susceptibility $\chi_{i j}$ even in the presence of local moments and/or high magnetic fields, provided that one knows the local moment (or magnetization) on each site for a given local configuration of atoms. Because it would not be computationally feasible to calculate those moments for concentrated alloys using the techniques of self-consistent 
alloy band theory, a new, computationally feasible scheme for their calculation is presented in Sec. III, thus making the formalism of Sec. II usable in practice.

In Sec. IV the formalism of Secs. II and III is applied to the case of three different local clusters of $\mathrm{Ni}$ and $\mathrm{Pd}$ atoms embedded in pure $\mathrm{Pd}$ and in exchange-enhanced effective media. First, the unenhanced local band susceptibilities $\Gamma_{i j}\left(\left\{\mu_{k}\right\}\right)$ are calculated as functions of the local moments $\mu_{k}$; then, the thermodynamic equilibrium local moments $\tilde{\mu}_{k}$ are determined self-consistently using the formalism of Sec. III. Finally, the resultant local exchange-enhanced susceptibilities $\chi_{i j}\left(\left\{\tilde{\mu}_{k}\right\}\right)$ are calculated and the change, $\delta \chi(0)$, induced in the uniform static susceptibility, $\bar{\chi}(0)$, of the different effective media is found for each cluster.

In order to calculate the spontaneous magnetization and the susceptibility of any alloy containing magnetic moments which depend strongly on their local environments, one must determine the moments $\tilde{\mu}_{k}$ and local susceptibilities $\chi_{i j}\left(\left\{\tilde{\mu}_{k}\right\}\right)$ for a large number $\left(\gtrsim 10^{4}\right)$ of different configurations of atoms on clusters containing 30 or more atoms. Because that would be very time consuming using calculations such as those presented in Sec. IV, we have developed simple, physically motivated interpolation formulas for the magnetization and local susceptibility of any cluster embedded in an effective medium as a function of $\bar{\chi}(0)$ and of the change, $\delta \chi_{p}(0)$, which would be induced in $\bar{\chi}(0)$ by that cluster in the absence of magnetic moments and which is easily calculated using the formalism of I. These interpolation formulas are presented in Sec. V. The parameters in those formulas can be determined by fitting to the results of self-consistent calculations such as those presented in Sec. IV. Finally, a brief summary and statement of conclusions are presented in Sec. VI.

\section{DERIVATION OF BASIC EQUATION FOR $\chi_{i j}(\omega)$}

The basic integral equation [Eq. (1.5) of I] which serves as the basic starting point for all RPA-cluster calculations of the susceptibility has previously been derived only for the paramagnetic regime and only within the approximation of linear response. We show here that an equation of the same form holds even in the presence of spontaneous or induced local moments. The derivation given here is similar to that given in Appen$\operatorname{dix} \mathbf{A}$ of I for the simpler case of linear response in the absence of spontaneous moments.

One starts from the same basic equations, Eq. (A1), (A2), and (A4), as in Appendix A of I. However, the presence of spontaneous local moments breaks crystalline symmetry so that the expectation value,

$$
\left\langle S_{i m}\right\rangle \equiv \bar{S}_{i m}+\delta\left\langle S_{i m}\right\rangle,
$$

of the $z$ component of the spin associated with the orbital $m$ on the site $i$ has a nonzero value, $\bar{S}_{i m}$, even in the absence of an applied magnetic field. Consequently, the expectation value,

$$
\left\langle n_{i m \sigma}\right\rangle \equiv \bar{n}_{i m \sigma}+\delta\left\langle n_{i m \sigma}\right\rangle,
$$

of the number operator $n_{i m \sigma}$ contains a part,

$$
\bar{n}_{i m \sigma}=\frac{1}{2} \bar{n}_{i m}+\operatorname{sgn}\{\sigma\} \bar{S}_{i m},
$$

which depends on the sign of $\sigma$ even in the absence of an external field. Also, the quantity $\bar{n}_{i m}=\bar{n}_{i m \uparrow}+\bar{n}_{i m \downarrow}$ can depend on the magnitude of the local magnetization, and hence on the applied field, through charge-transfer effects. Thus, the effective potential $V_{i m}^{\mathrm{HF}}$ in the Hartree-Fock (HF) one-electron Hamiltonian (A5) of $I$ is replaced by the spin-dependent potential

$$
V_{i m \sigma}^{\mathrm{HF}}=\varepsilon_{i m}+\frac{1}{2} U_{i}^{m m} \delta\left\langle n_{i m}\right\rangle+\sum_{m^{\prime}}\left\{U_{i}^{m m^{\prime}} \bar{n}_{i m^{\prime} \bar{\sigma}}+\left(1-\delta_{m m^{\prime}}\right)\left[\left(U_{i}^{m m^{\prime}}-J_{i}^{m m^{\prime}}\right) \bar{n}_{i m^{\prime} \sigma}+\left(U_{i}^{m m^{\prime}}-\frac{1}{2} J_{i}^{m m^{\prime}}\right) \delta\left\langle n_{i m^{\prime}}\right\rangle\right]\right\},
$$

where $\bar{\sigma}=-\sigma$. The equation for the local effective field, $h_{i m}^{\text {eff }}$, is unchanged except for the replacement of $\left\langle S_{i m}\right\rangle$ by $\delta\left\langle S_{i m}\right\rangle$ (the two are equal in the absence of local moments). For the model case of five equivalent $d$ subbands, the one-electron Hartree-Fock Hamiltonian assumes the form

$$
\begin{aligned}
H^{\mathrm{HF}}= & \sum_{i, j, \sigma} t_{i j} c_{i \sigma}^{\dagger} c_{j \sigma}+\sum_{i, \sigma} V_{i \sigma}^{\mathrm{HF}} n_{i \sigma} \\
& -\frac{1}{2} \sum_{i} h_{i}^{\mathrm{eff}}\left(n_{i \uparrow}-n_{i \downarrow}\right) e^{i \omega t},
\end{aligned}
$$

where

$$
\begin{aligned}
& V_{i \sigma}^{\mathrm{HF}}=V_{i \sigma}^{(0)}+\frac{1}{2} v_{i} \delta\left\langle n_{i}\right\rangle e^{-i \omega t}, \\
& V_{i \sigma}^{(0)}=\varepsilon_{i}+\frac{1}{2} v_{i} \bar{n}_{i}-\operatorname{sgn}\{\sigma\} u_{i} \bar{S}_{i},
\end{aligned}
$$

$$
h_{i}^{\mathrm{eff}}=h_{i}+2 u_{i} \delta\left\langle S_{i}\right\rangle e^{-i \omega t}
$$

the potentials $v_{i}$ and $u_{i}$ are given by $\left(9 U_{i}-4 J_{i}\right) / 5$ and $\left(U_{i}+4 J_{i}\right) / 5$, respectively, and the units $\left(g \mu_{B}\right)^{2}=2$ are used for convenience.

From Eqs. (2.5)-(2.8), the incremental field-induced changes in the local energy levels $\varepsilon_{k \sigma}$ are given by

$$
\delta \varepsilon_{k \sigma}=\frac{1}{2} v_{k} \delta\left\langle n_{k}\right\rangle-\frac{1}{2} \operatorname{sgn}\{\sigma\}\left[h_{k} \exp (i \omega t)+2 u_{k} \delta\left\langle S_{k}\right\rangle\right] .
$$

Thus, because $\left\langle n_{i \sigma}(\omega)\right\rangle$ can be expressed within the RPA solely as a function of the energies $\varepsilon_{k \sigma}(\omega)$ for the same spin $\sigma$ and frequency $\omega$, one finds the result

$$
d\left\langle n_{i \sigma}(\omega)\right\rangle / d h_{j}=e^{i \omega t} \sum_{k} \Gamma_{i k}^{(0)}(\omega)\left\{\left[\delta_{k j}+u_{k} \chi_{k j}(\omega)\right] \operatorname{sgn}\{\sigma\}-v_{k} \Lambda_{k j}(\omega)\right\}
$$


where

$$
\begin{aligned}
& \Gamma_{i k \sigma}^{(0)}(\omega) \equiv-\frac{1}{2} \partial\left\langle n_{i \sigma}(\omega)\right\rangle / \partial \varepsilon_{k \sigma}(\omega), \\
& \chi_{k j}(\omega) \equiv 2 e^{-i \omega t} d\left\langle S_{k}\right\rangle / d h_{j},
\end{aligned}
$$

and

$$
\Lambda_{k j}(\omega) \equiv e^{-i \omega t} d\left\langle n_{k}(\omega)\right\rangle / d h_{j} .
$$

Upon evaluating Eq. (2.10) for spin up and for spin down and adding and subtracting the resultant equations, one finds the pair of coupled equations,

$$
\begin{aligned}
\Lambda_{i j}(\omega)=\Lambda_{i j}^{(0)}(\omega)+\sum_{k} & {\left[\Lambda_{i k}^{(0)}(\omega) u_{k} \chi_{k j}(\omega)\right.} \\
& \left.-\Gamma_{i k}^{(0)}(\omega) v_{k} \Lambda_{k j}(\omega)\right],
\end{aligned}
$$

and

$$
\begin{aligned}
\chi_{i j}(\omega)=\Gamma_{i j}^{(0)}(\omega)+\sum_{k}[ & \Gamma_{i k}^{(0)}(\omega) u_{k} \chi_{k j}(\omega) \\
& \left.-\Lambda_{i k}^{(0)}(\omega) v_{k} \Lambda_{k j}(\omega)\right],
\end{aligned}
$$

where

$$
\Lambda_{i j}^{(0)}(\omega) \equiv \sum_{\sigma} \Gamma_{i j \sigma}^{(0)}(\omega) \operatorname{sgn}\{\sigma\}
$$

and

$$
\Gamma_{i j}^{(0)}(\omega) \equiv \sum_{\sigma} \Gamma_{i j \sigma}^{(0)}(\omega)
$$

Upon solving Eqs. (2.12) and (2.13) simultaneously, one finds the equation

$$
\chi_{i j}(\omega)=\Gamma_{i j}(\omega)+\sum_{k} \Gamma_{i k}(\omega) u_{k} \chi_{k j}(\omega),
$$

where

$$
\begin{aligned}
\Gamma_{i j}(\omega)= & \Gamma_{i j}^{(0)}(\omega) \\
& -\sum_{k, l} \Lambda_{i k}^{(0)}(\omega) v_{k}\left\{\left[1+\Gamma^{(0)}(\omega) \mathbf{v}\right]^{-1}\right\}_{k l} \Lambda_{l j}^{(0)}(\omega)
\end{aligned}
$$

differs from the local band susceptibility $\Gamma_{i j}^{(0)}(\omega)$ only if the application of a magnetic field on site $j$ induces a charge transfer on site $i$, i.e., only if substantial moments exist on sites $i$ and $j$ or on sites in the immediate vicinity of sites $i$ and $j$. Equation (2.15) is identical in form to Eq. (1.5) of I, which forms the basic starting point for that paper. Thus, all of the formalism of $I$ is applicable even for very large magnetic fields and even in the presence of local moments, provided that one can calculate the susceptibilities $\Gamma_{i j}(\omega)$ defined by Eq. (2.16).

Because $\Gamma_{i j}^{(0)}$ and $\Lambda_{i j}^{(0)}$ are spatially short ranged, very nearly vanishing for sites $i$ and $j$ separated by more than a third- or fourth-nearest-neighbor distance, the dimension of the matrix to be inverted in Eq. (2.16) for $\Gamma_{i j}(\omega)$ is not too large, typically less than, or of the order of, 50. Thus, the susceptibilities $\Gamma_{i j}$ can be found easily once the moments $\mu_{k}$ in the vicinity of sites $i$ and $j$ are known and once the local band susceptibilities
$\Gamma_{i j \sigma}^{(0)}\left(\left\{\mu_{k}\right\}\right)$ are known as functions of the $\mu_{k}$. From the fluctuation-dissipation theorem, the $\Gamma_{i j \sigma}^{(0)}$ are given by

$\Gamma_{i j \sigma}^{(0)}(\omega)=(2 \pi)^{-1} \operatorname{Im} \int_{-\infty}^{\varepsilon_{F}} d \varepsilon G_{i j \sigma}^{(0)}(\varepsilon) G_{i j \sigma}^{(0)}(\varepsilon+\omega)$,

where the Green's functions $G_{i j \sigma}^{(0)}$ are calculated by band theory in the presence of the local moments $\mu_{k}$. Unfortunately the presence of local moments considerably complicates the calculation of the $G_{i j \sigma}^{(0)}$; even for a known distribution of moments $\mu_{k}$, it becomes a self-consistent iterative calculation. The self-consistent simultaneous calculation of the $\mu_{k}$ and the $G_{i j \sigma}^{(0)}$ by band-theoretic techniques is an awesome task for other than very small clusters. Thus, in the next section we develop a new, more easily applied theory for the self-consistent calculation of the moments $\mu_{k}$ and the local susceptibilities $\chi_{i j}\left(\left\{\mu_{k}\right\}\right)$.

\section{SELF-CONSISTENT SUSCEPTIBILITY FORMULATION FOR THE DETERMINATION OF LOCAL MOMENTS AND SUSCEPTIBILITIES}

In this section a new formalism is developed for the self-consistent determination of the equilibrium local moments $\tilde{\mu}_{k}$ and the exchange-enhanced susceptibilities $\chi_{i j}\left(\omega ;\left\{\tilde{\mu}_{k}\right\}\right)$, for a cluster embedded in an exchangeenhanced host or effective medium. The formalism developed here is not as well suited as is that of spinpolarized self-consistent band theory $9,10,12$ for determining very accurately the local magnetization of isolated magnetic impurities. However, it is better suited to the calculation of the local magnetization of concentrated exchange-enhanced magnetic alloys and is the first formalism ever developed for the susceptibility of such alloys.

Because the local band susceptibilities, $\Gamma_{i j}\left(\omega ;\left\{\mu_{k}\right\}\right)$, are functions of the moments $\mu_{k}$ on all sites $k$ in the vicinity of sites $i$ or $j$, the theory to be developed must allow for the existence of general off-diagonal disorder. Thus, as in Sec. II C of paper I, for the case of an $n$-site cluster, $C$, embedded in an exchange-enhanced host or effective medium, one must allow $\Gamma_{i j}$ to differ from the local susceptibility, $\bar{\Gamma}_{i j}$, of the host or effective medium in which it is embedded for all sites $i$ and $j$ which lie in an $n+n^{\prime}+n^{\prime \prime}$ site cluster, $C^{\prime}$, containing the cluster $C$, all sites in the host or effective medium having substantial induced moments and all sites connected to those sites by nonzero matrix elements, $\Gamma_{k l}$. This means that in the formalism of this section all matrices to be inverted must be of dimension $n+n^{\prime}+n^{\prime \prime}$, not just of dimension $n$.

In the presence of applied fields, $H_{k}$, parallel to the $z$ axis on each site $k$, the equilibrium values, $\widetilde{u}_{k}$, for the $z$ components of the moments $\mu_{k}$ can be formally determined by minimizing the Gibbs free energy with the fields $H_{k}$ and the temperature $T$ held fixed. This formal procedure yields two possible equilibrium conditions for the entire system at zero temperature: either

$$
\left[\partial E\left(\left\{\mu_{k}\right\}\right) / \partial \mu_{i}\right]_{\left\{\mu_{k}\right\}=\left\{\tilde{\mu}_{k}\left(\left\{H_{k}\right\}\right)\right\}}=H_{i}
$$


for all $i$, or, by symmetry in the absence of external fields,

$$
\left[\partial E\left(\left\{\mu_{k}\right\}\right) / \partial \mu_{i}\right]_{\left\{\mu_{k}\right\}=\{0\}}=0
$$

for all $i$, where $E$ is the total energy of the system. Of course, for any system containing spontaneous moments Eq. (3.1b) yields only an unstable local minimum in $E$. Upon subtracting Eq. (3.1b) from Eq. (3.1a), one can write the resultant expression in the integral form

$$
\sum_{j=1}^{n+n^{\prime}} \int_{0}^{\tilde{\mu}_{j}\left(\left\{H_{k}\right\}\right)} d \mu_{j} \partial^{2} E\left(\left\{\mu_{k}\right\}\right) / \partial \mu_{i} \partial \mu_{j}=H_{i}
$$

where

$$
\mu_{k}=\left\{\begin{array}{l}
\tilde{\mu}_{k}\left(\left\{H_{k}\right\}\right) \text { for } k>j, \\
0 \text { for } k<j
\end{array}\right.
$$

This expression, although it appears to be more complicated than Eqs. (3.1), is very useful in determining the zero-temperature magnetization because at zero temperature

$$
\partial^{2} E\left(\left\{\mu_{k}\right\}\right) / \partial \mu_{i} \partial \mu_{j}=\left[\chi^{-1}\left(\left\{\mu_{k}\right\}\right)\right]_{i j}
$$

Upon substituting Eq. (3.4) into Eq. (3.2) and using the relationship

$$
\left[\boldsymbol{X}^{-1}\left(\left\{\mu_{k}\right\}\right)\right]_{i j}=\left[\Gamma^{-1}\left(\left\{\mu_{k}\right\}\right)\right]_{i j}-u_{i} \delta_{i j},
$$

which follows from Eq. (2.15), one finds the set of equations

$$
\sum_{j=1}^{n+n^{\prime}} \int_{0}^{\tilde{\mu}_{j}\left(\left\{H_{k}\right\}\right)} d \mu_{j}\left[\Gamma^{-1}\left(\left\{\mu_{k}\right\}\right)\right]_{i j}=H_{i}+u_{i} \tilde{\mu}_{i}\left(\left\{H_{k}\right\}\right)
$$

for the equilibrium moments $\tilde{\mu}_{k}$, where the moments $\mu_{k}$ are given by Eq. (3.3). For a cluster containing $r \leq n+n^{\prime}$ inequivalent sites, Eq. (3.6) constitutes a set of $r$ coupled nonlinear integral equations to be solved selfconsistently for the set of $r$ inequivalent local moments, $\tilde{\mu}_{k}$. For the special case of a pure material subjected to a uniform magnetic field $H$, Eqs. (3.6) reduce to the single equation

$$
\int_{0}^{\tilde{\mu}(H)} d \mu \Gamma^{-1}(0 ; \mu)=H+u \tilde{\mu}(H)
$$

where

$$
\Gamma^{-1}(0 ; \mu)=N\left(\sum_{i, j} \Gamma_{i j}(\mu)\right]^{-1}
$$

is the inverse of the uniform static band susceptibility.

The exchange-enhanced local susceptibilities, $\chi_{i j}\left(\left\{\tilde{\mu}_{k}\right\}\right)$ can also be determined from Eq. (3.6) for $\omega=0$ (or for any $\omega$ sufficiently small that the local moments follow the applied fields $H_{i}$ adiabatically). Upon differentiating Eq. (3.6) with respect to $H_{m}$, one finds the equation

$$
\begin{array}{r}
\sum_{j=1}^{n+n^{\prime}} \chi_{j m}\left(\left\{\tilde{\mu}_{k}\right\}\right)\left\{\left[\Gamma^{-1}\left(\left\{\tilde{\mu}_{k}\right\}\right)\right]_{i j}+\phi_{i j}\left(\left\{\tilde{\mu}_{k}\right\}\right)\right\} \\
=\delta_{i m}+u_{i} \chi_{i m}\left(\left\{\tilde{\mu}_{k}\right\}\right),
\end{array}
$$

where $\mu_{k}$ is defined by Eq. (3.3) for $k \neq j$ and $\mu_{k}=\widetilde{\mu}_{j}$ for $k=j$, the matrix $\phi$ has matrix elements

$$
\phi_{i j}\left(\left\{\tilde{\mu}_{k}\right\}\right)=\sum_{l=j+1}^{n+n^{\prime}} \int_{0}^{\tilde{\mu}_{l}} d \mu_{l} \frac{\partial}{\partial \tilde{\mu}_{j}}\left[\Gamma^{-1}\left(\tilde{\mu}_{1}, \ldots, \tilde{\mu}_{l-1}, \mu_{l}, 0,0, \ldots, 0\right)\right]_{i l} \text {, }
$$

and $\mathbf{u}$ is a diagonal matrix with matrix elements $u_{i j}=u_{i} \delta_{i j}$. Upon writing Eq. (3.8) in matrix form, one can easily invert it to find the result

$$
\chi_{i j}\left(\left\{\tilde{\mu}_{k}\right\}\right)=\left\{\left[\boldsymbol{\Gamma}^{-1}\left(\left\{\tilde{\mu}_{k}\right\}\right)+\phi\left(\left\{\tilde{\mu}_{k}\right\}\right)-\mathbf{u}\right]^{-1}\right\}_{i j} .
$$

It then follows immediately that the change, $\delta \chi\left(0,\left\{\tilde{\mu}_{k}\right\}\right)$, introduced in the $\overrightarrow{\mathbf{q}}=0$ component of the exchangeenhanced static susceptibility, $\bar{\chi}(0)$, of a host or effective medium by embedding a cluster in it is given by the equation

$$
\delta \chi\left(0 ;\left\{\tilde{\mu}_{k}\right\}\right)=\chi\left(0 ;\left\{\tilde{\mu}_{k}\right\}\right)-\bar{\chi}(0)=N^{-1} \sum_{i=1}^{n+n^{\prime}}\left(\sum_{j=1}^{n+n^{\prime}+n^{\prime \prime}}\left\{\left[\Gamma^{-1}\left(\left\{\tilde{\mu}_{k}\right\}\right)+\phi\left(\left\{\tilde{\mu}_{k}\right\}\right)-\mathbf{u}\right]^{-1}\right\}_{i j}-\bar{\chi}(0)\right)
$$

It is assumed here that the local band susceptibilities $\Gamma_{i j}\left(\left\{\mu_{k}\right\}\right)$ are known at least approximately from model alloy cluster calculations performed for a set of fixed moment distributions. The inversion of the matrix $\Gamma$ for any cluster embedded in an effective medium is then straightforward using the techniques of Sec. II of I. We first express $\Gamma$ in the form

$$
\Gamma\left(\left\{\mu_{k}\right\}\right)=\bar{\Gamma}\left[1+\bar{\Gamma}^{-1} \delta \Gamma\left(\left\{\mu_{k}\right\}\right)\right]
$$

as a sum of the matrix band susceptibility, $\bar{\Gamma}$, of the medium in which the cluster is embedded and the matrix

$$
\delta \Gamma\left(\left\{\mu_{k}\right\}\right) \equiv \Gamma\left(\left\{\mu_{k}\right\}\right)-\bar{\Gamma}
$$


which has nonzero matrix elements $\delta \Gamma_{i j}$ only for sites $i$ and $j$ both of which are in the cluster $C^{\prime}$. Inverting Eq. (3.11) yields the result

$\boldsymbol{\Gamma}^{-1}\left(\left\{\boldsymbol{\mu}_{k}\right\}\right)=\overline{\boldsymbol{\Gamma}}^{-1}-\overline{\boldsymbol{\Gamma}}^{-1} \delta \boldsymbol{\Gamma}\left(\left\{\mu_{k}\right\}\right) \boldsymbol{\Gamma}^{-1}\left(\left\{\boldsymbol{\mu}_{k}\right\}\right)$,

which is analogous to Eq. (2.6) of I and thus can be solved by the same procedure. One finds that it has the solution

$$
\begin{aligned}
\Gamma^{-1}\left(\left\{\mu_{k}\right\}\right)=\overline{\boldsymbol{\Gamma}}^{-1}\{1- & \delta \Gamma\left(\left\{\mu_{k}\right\}\right) \\
& \left.\times\left[1+\overline{\boldsymbol{\Gamma}}-1 \delta \Gamma\left(\left\{\mu_{k}\right\}\right)\right]^{-1} \overline{\boldsymbol{\Gamma}}^{-1}\right\}
\end{aligned}
$$

or, in site representation,

$$
\left[\boldsymbol{\Gamma}^{-1}\left(\left\{\mu_{k}\right\}\right)\right]_{i j}=\left(\overline{\boldsymbol{\Gamma}}^{-1}\right)_{i j}-\sum_{\substack{l, m, n \varepsilon \mathcal{C}^{\prime}}}\left(\overline{\boldsymbol{\Gamma}}^{-1}\right)_{i l} \delta \Gamma_{l m}\left(\left\{\mu_{k}\right\}\right)\left\{\left[1+\overline{\boldsymbol{\Gamma}}^{-1} \delta \boldsymbol{\Gamma}\left(\left\{\boldsymbol{\mu}_{k}\right\}\right)\right]^{-1}\right\}_{m n}\left(\overline{\boldsymbol{\Gamma}}^{-1}\right)_{n j}
$$

where $\overline{\boldsymbol{\Gamma}}^{-1}$ is found by taking the Fourier transform of $\overline{\boldsymbol{\Gamma}}^{-1}(\overrightarrow{\mathbf{q}})$. For any cluster, the use of Eqs. (3.12) and (3.14) in Eqs. (3.6) - (3.9) completes the determination of the zero-temperature equilibrium local moments $\tilde{\mu}_{k}$, the exchange-enhanced local susceptibilities $\chi_{i j}\left(\left\{\tilde{\mu}_{k}\right\}\right)$ and the quantity $\delta \chi\left(0 ;\left\{\tilde{\mu}_{k}\right\}\right)$ in terms of the unenhanced local susceptibilities $\Gamma_{i j}\left(\left\{\tilde{\mu}_{k}\right\}\right)$ and the band susceptibility $\bar{\Gamma}(\overrightarrow{\mathbf{q}})$ of the host or effective medium in which the cluster is embedded.

\section{SAMPLE NUMERICAL CALCULATIONS OF LOCAL MOMENTS AND CLUSTER MAGNETIZATIONS AND SUSCEPTIBILITIES}

In this section we present sample calculations of the nucleating local magnetic moments, the magnetization density and total cluster magnetization, the local susceptibilities $\chi_{i j}\left(\left\{\tilde{\mu}_{k}\right\}\right)$ and the quantity $\delta \chi\left(0 ;\left\{\tilde{\mu}_{k}\right\}\right)$ for three simple magnetic configurations of $\mathrm{Ni}$ atoms embedded in $\mathrm{Pd}$ and in effective media representative of dilute $P d-\mathrm{Ni}$ alloys. The derivation of the parameters used in these calculations will be presented along with our complete results for the magnetization and susceptibility of $\mathrm{Pd}-\mathrm{Ni}$ alloys in paper IV. For pure Pd the uniform static band susceptibility, $\widetilde{\Gamma}(0)$, was chosen to be 1.2 states/eV atom of which 0.9808 states/eV atom were associated with the $d$ band and, hence, were to be exchange enhanced. The choice of the value 11.2 states/eV atom for the total exchange-enhanced susceptibility, $\widetilde{\chi}(0)$, of Pd then led to the value $0.9285 \mathrm{eV}$ for the intraatomic Coulomb repulsion, $\tilde{u}^{(d)}$, on Pd atoms.

The values of the matrix elements $\widetilde{\Gamma}_{i j}^{(d)}$ of the susceptibility of Pd were required to satisfy the sum rule

$$
\sum_{j} \widetilde{\Gamma}_{i j}^{(d)}=\widetilde{\Gamma}^{(d)}(0)=0.9808 \text { states } / \mathrm{eV} \text { atom }
$$

and to yield an exchange-enhanced susceptibility of the form

$$
\tilde{\chi}(q)=\kappa^{2} \widetilde{\chi}(0) /\left(\kappa^{2}+q^{2}\right)
$$

determined by neutron-scattering experiments, ${ }^{13}$ with $\kappa=0.3 \AA^{-1}$. For simplicity of notation, throughout the remainder of this paper, the superscript in $\widetilde{\Gamma}_{i j}^{(d)}$ and $\widetilde{u}^{(d)}$ is omitted. Although Eqs. (4.1) and (4.2) do not suffice to determine the $\widetilde{\Gamma}_{i j}$ uniquely, it was found that different approximations for the $\widetilde{\Gamma}_{i j}$ consistent with Eqs. (4.1) and (4.2) all yield the same final results within $3-4 \%$. Four sets of values of the $\widetilde{\Gamma}_{i j}$ are shown in Table I; there, $\widetilde{\Gamma}_{n}$ represents the value of $\widetilde{\Gamma}_{i j}$ for any pair of sites which are $n$th nearest neighbors of one another. The third set was used in the calculations reported here; the other sets give very similar results.

Values of the band susceptibilities $\Gamma_{i j}^{(0)}\left(\left\{\mu_{k}\right\}\right)$ were determined from model tight-binding band calculations for magnetic clusters of three atoms embedded in unmagnetized Pd. The results for the diagonal matrix elements $\Gamma_{i i \sigma}^{\mathrm{Ni}}$ on $\mathrm{Ni}$ sites were expressed as a function solely of the moment $\mu_{i}$ on that site. The matrix elements $\Gamma_{i j \sigma}^{\mathrm{Ni}-\mathrm{Ni}}$ and $\Gamma_{i j \sigma}^{\mathrm{Pd}-\mathrm{Ni}}$ for nearest-neighbor pairs of atoms were expressed solely as a function of the moments on the sites $i$ and $j$. Finally, the matrix elements $\Gamma_{i j \sigma}^{\mathrm{Pd}-\mathrm{Pd}}$ for nearest-neighbor $\mathbf{P d}$ atoms were expressed as a function of the moments on the sites $i$ and $j$ and of the moment of the third site of the cluster. The values of $\Gamma_{i j}$ were then calculated from Eq. (2.16); they were found to be almost independent of the value chosen for the potentials $v_{i}$ used in calculating charge transfer, within reason. The value $v_{i}=3.24 \mathrm{eV}$ was used for both $\mathrm{Pd}$ and $\mathrm{Ni}$ atoms. The parameters of the band structure were chosen so as to give the correct values for $\widetilde{\Gamma}(0)$ and $\widetilde{\chi}(0)$, the correct inverse range, $\kappa$, of $\widetilde{\chi}(q)$, the correct number of $d$ holes for Pd and the correct saturation moment, $1 \mu_{B},{ }^{14}$ for $\mathrm{Ni}$ atoms in dilute $P d-\mathrm{Ni}$ alloys. Finally, the results of these calculations were generalized by assuming the ratios $\Gamma_{i j \sigma}\left(\mu_{i}, \mu_{j}\right) / \Gamma_{i j \sigma}(0,0)$ to be the same for nearest and second-nearest neighbors, by invoking the geometric approximation,

$$
\left(\Gamma_{i j \sigma}^{\mathrm{Pd}-\mathrm{Ni}}\right)^{2}=\Gamma_{i j \sigma}^{\mathrm{Pd}-\mathrm{Pd}} \Gamma_{i j \sigma}^{\mathrm{Ni}-\mathrm{Ni}},
$$

\begin{tabular}{|c|c|c|c|c|}
\hline $\begin{array}{c}\text { Parameter } \\
\text { set }\end{array}$ & $\widetilde{\Gamma}_{0}$ & $\begin{array}{l}\widetilde{\Gamma}_{1} \\
\text { (state }\end{array}$ & $\begin{array}{r}\tilde{\Gamma}_{2} \\
\text { atom) }\end{array}$ & $\widetilde{\Gamma}_{3}$ \\
\hline 1 & 0.2791 & 0.0513 & 0.00795 & 0.0016 \\
\hline 2 & 0.2584 & 0.0520 & 0.01640 & 0 \\
\hline 3 & 0.1948 & 0.0611 & 0.00880 & 0 \\
\hline 4 & 0.1372 & 0.0703 & 0 & 0 \\
\hline
\end{tabular}

and by averaging over the moments on all of the nearest neighbors of sites $i$ and $j$ in calculating $\Gamma_{i j \sigma}^{\mathrm{Pd}-\mathrm{Pd}}$. To illus-

TABLE I. Values of the single-spin local band susceptibilities, $\widetilde{\Gamma}_{i j} \equiv \widetilde{\Gamma}_{n}$, for sites $i$ and $j$ which are $n$th nearest neighbors in pure $\mathbf{P d}$. 
trate our results for the moment dependence of the band susceptibilities $\Gamma_{i j}\left(\mu_{i}, \mu_{j}\right)$, we show in Fig. 1 the moment dependencies of $\Gamma_{i i}^{\mathrm{Ni}}\left(\mu_{i}\right)$ and of $\Gamma_{i j}^{\mathrm{Ni}-\mathrm{Ni}}\left(\mu_{i}, \mu_{j}\right)$ for nearest-neighbor sites $i$ and $j$ having equal moments.

The two primary possible effects responsible for the enhancement of the local susceptibility and/or the formation of local moments on impurity atoms or clusters in an exchange-enhanced effective medium are

(i) an increased Coulomb integral, $u_{i}$, on the impurity sites, and

(ii) an increase in $\Gamma_{i j}$ if either one or both of the sites $i$ and $j$ are impurity sites.

In previous treatments of exchange-enhanced alloy susceptibilities, for simplicity the second effect has usually been neglected. However, in $\mathrm{Pd}-\mathrm{Ni}$ alloys the second effect is very large because the value of the site spectralweight function, $A_{i}(\omega)$, is much greater at the Fermi level for $\mathrm{Ni}$ sites than for Pd sites, owing to the very small number of $d$ holes in Pd. Our calculations, to be reported in paper IV, indicate that the concentration dependence of the susceptibility and magnetization of Pd-Ni alloys can be explained almost entirely on the basis of the second effect, with no enhancement of the Coulomb integral on $\mathrm{Ni}$ sites. Thus, in the numerical calculations presented here any possible enhancement of $u_{i}$ on Ni sites is neglected.

For a magnetic cluster containing $n$ sites grouped into $r \leq n$ shells or partial shells, with the moment on every atom in any given shell, $\alpha$, being the same by symmetry, the numerical calculation of the spontaneous local moments $\tilde{\mu}_{\alpha}$ on the atoms of each shell is performed iteratively as follows. First, one chooses an initial set of moments, $\left\{\tilde{\mu}_{\alpha}^{(0)}\right\}$, and determines the resultant cluster band-susceptibility matrices $\Gamma\left(\left\{\mu_{\alpha}\right\}\right)$ and $\Gamma^{-1}\left(\left\{\mu_{\alpha}\right\}\right)$ from Eqs. (2.16) and (3.14), respectively. Then, by fitting the $\left[\Gamma^{-1}\left(\left\{\mu_{\alpha}\right\}\right)\right]_{i j}$ to even multinomials in the $\mu_{\alpha}$ 's, one can integrate Eq. (3.6) analytically. Upon solving the resultant set of $r$ coupled nonlinear algebraic equations, one then obtains a new set of moments, $\tilde{\mu}_{\alpha}$. This pro-

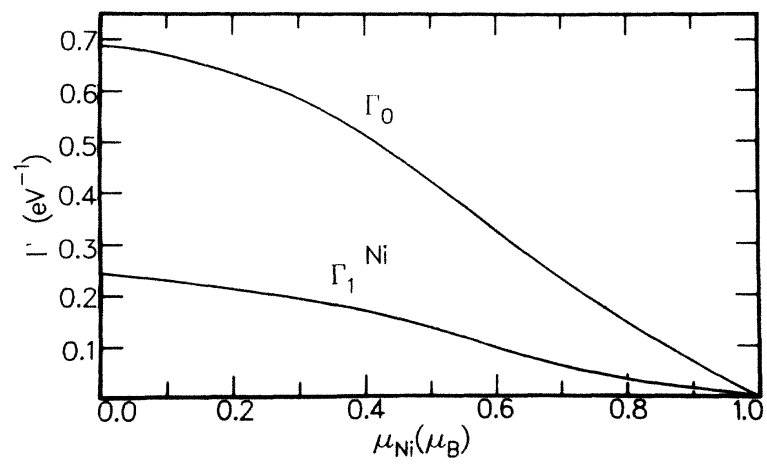

FIG. 1. Local diagonal, $\Gamma_{0}^{\mathrm{Ni}}$, and off-diagonal, $\Gamma_{1}^{\mathrm{Ni}-\mathrm{Ni}}$, band susceptibilities as functions of the $\mathrm{Ni}$ moment, for a cluster of three nearest-neighbor $\mathrm{Ni}$ impurities with the same moment embedded in the fcc Pd lattice, with $v=3.24 \mathrm{eV}$. cedure is then repeated iteratively until one attains selfconsistency.

In principle, one can determine the spontaneous moments on every atom of any cluster by following this iterative numerical procedure. However, in practice the highly nonlinear nature of the equations to be solved simultaneously, the large number of shells in a cluster, typically $r \gtrsim 10$, and the existence of different selfconsistent solutions which correspond only to local minima in the total energy lead to serious numerical difficulties. We have found that nonlinearities and saturation effects are important only for those $\mathrm{Ni}$ atoms which nucleate a magnetic cluster or polarization cloud and for the $\mathrm{Pd}$ atoms which are first- or second-nearest neighbors to those $\mathrm{Ni}$ atoms. Thus, we have used the nonlinear Eqs. (3.6) and (3.9) to calculate the spontaneous moments $\tilde{\mu}_{i}$ and the uniform-field susceptibilities $\chi_{i} \equiv \Sigma_{j} \chi_{i j}$ only for those sites. For all other shells within the cluster, the spontaneous local moments and uniform-field susceptibilities were calculated within linear response theory from the equations

$$
\tilde{\mu}_{i}=\sum_{j} \Gamma_{i j}\left(\left\{\tilde{\mu}_{k}\right\}\right) u_{j} \tilde{\mu}_{j}
$$

and

$\chi_{i}\left(\left\{\tilde{\mu}_{k}\right\}\right)=\sum_{j}\left\{\left[1-\Gamma\left(\left\{\tilde{\mu}_{k}\right\}\right) \mathbf{u}\right]^{-1}\right\}_{i j} \Gamma_{j}\left(\left\{\tilde{\mu}_{k}\right\}\right)$,

respectively, where $\Gamma_{j} \equiv \sum_{l} \Gamma_{j l}$. Beyond the cluster, for the shells with $\alpha>r$, the effects of asymmetry were neglected and the moments and uniform-field susceptibilities in the embedding effective medium were approximated by the Yukawa expressions ${ }^{14}$

$$
\tilde{\mu}_{\alpha}=\bar{\mu}_{0}(H=0) R_{\alpha}^{-1} \exp \left(-\bar{\kappa} R_{\alpha}\right)
$$

and

$$
\chi_{\alpha}\left(\left\{\tilde{\mu}_{k}\right\}\right)=\left[d \bar{\mu}_{0}(H) / d H\right] R_{\alpha}^{-1} \exp \left(-\bar{\kappa} R_{\alpha}\right)+\bar{\chi}(0),
$$

respectively. Here, $R_{\alpha}$ is the distance of the $\alpha$ th shell from the center of the cluster, $\bar{\kappa}$ was determined by fitting the susceptibility of the effective-medium to the form

$$
\bar{\chi}(\overrightarrow{\mathbf{q}})=\bar{\kappa}^{2} \bar{\chi}(0) /\left(\bar{\kappa}^{2}+q^{2}\right),
$$

and $\bar{\mu}_{0}(H=0)$ then was determined from a least-squares fit of Eq. (4.5) to the local moments on the outer shells of the cluster. The derivative $d \bar{\mu}_{0}(H) / d H$ was evaluated using the expression

$$
\frac{d \bar{\mu}_{0}(H)}{d H}=\frac{\sum_{\beta} Z_{\beta} R_{\beta}^{-1} \exp \left(-\bar{\kappa} R_{\beta}\right)\left[\chi_{\beta}\left(\left\{\tilde{\mu}_{k}\right\}\right)-\bar{\chi}(0)\right]}{\sum_{\beta} Z_{\beta} R_{\beta}^{-2} \exp \left(-2 \bar{\kappa} R_{\beta}\right)}
$$

where the sums over $\beta$ extend over all shells in the cluster for which the linear Eqs. (4.3) and (4.4) were used, and $Z_{\beta}$ is the number of sites in the $\beta$ th shell.

The total giant moment associated with the cluster 
was then calculated using the formula

$\mu=\sum_{\alpha=1}^{r} Z_{\alpha} \tilde{\mu}_{\alpha}+\left[16 \pi \bar{\mu}_{0}(H=0) / a^{3}\right] \int_{R_{n}}^{R_{N}} d r r \exp (-\bar{\kappa} r)$,

where the second term gives that part of the total moment which arises from the polarization of the effective medium in which the cluster is embedded. Here, $a=3.889 \AA$ is the lattice constant of $\mathrm{Pd}$,

$$
R_{n}=\left(Z_{r+1} R_{r}+Z_{r} R_{r+1}\right) /\left(Z_{r}+Z_{r+1}\right)
$$

is the radius of the embedded cluster, and $R_{N}$ is the effective radius of the polarization cloud. The actual magnetic polarization clouds follow the Yukawa form (4.5) even approximately only for a few angstroms. The nonanalyticity in $\Gamma(\overrightarrow{\mathbf{q}})$ which gives rise to oscillations in $\Gamma(\overrightarrow{\mathbf{r}})$ also gives rise to oscillations in $\chi(\overrightarrow{\mathbf{r}})$ and, hence, to an effective cutoff radius $R_{N}$ for magnetic polarization clouds in $P d$-Ni alloys. The cutoff radius $R_{N}$ was calculated to be approximately $9.0 \AA$ by using percolation theory to fit the experimentally observed ${ }^{15}$ critical concentration of magnetic polarization clouds for ferromagnetism. That cutoff radius corresponds to a polarization cloud containing $N \simeq 210$ atoms. Finally, the change, $\delta \chi\left(0 ;\left\{\tilde{\mu}_{k}\right\}\right)$, induced in the static uniform exchange-enhanced susceptibility, $\bar{\chi}(0)$, of the effective medium by embedding the cluster was calculated from Eq. (3.10).

To illustrate the type of results obtained using the numerical procedure described above, we present here the results of model calculations performed for each of three simple clusters, each embedded in pure $\mathrm{Pd}$ and in various effective media. The first cluster consists of three nearest-neighbor $\mathrm{Ni}$ atoms together with $\mathrm{Pd}$ atoms on the 44 sites (12 shells) which are first or second neighbors to any of the Ni sites. The second consists of three neighboring $\mathrm{Ni}$ atoms arranged in a straight line together with $\mathrm{Pd}$ atoms on the 30 atoms (seven shells) which are first or second neighbors to the central $\mathrm{Ni}$ site and/or nearest neighbors to one of the end $\mathrm{Ni}$ sites. The third consists of four nearest-neighbor $\mathrm{Ni}$ atoms together with Pd atoms on the 40 sites (but only four inequivalent shells) which are first or second neighbors to any of the $\mathrm{Ni}$ sites.

We found moments of approximately $0.4 \mu_{B}, 0.4 \mu_{B}$, and $0.6 \mu_{B}$, respectively, on the central $\mathrm{Ni}$ atoms in these three clusters, depending only weakly on the embedding medium for the clusters, in agreement with the value $0.5 \mu_{B}$ found experimentally by Chouteau ${ }^{16}$ for the average moment on the $\mathrm{Ni}$ atoms in such clusters and a factor of 2 smaller than the saturation moment of $1 \mu_{B}$ suggested by the neutron-scattering data of Cable and Child. ${ }^{14}$ Because the $\mathrm{Ni}$ atoms in the first and third clusters are arranged in central compact subclusters, the zero-temperature equilibrium local moments, $\tilde{\mu}_{n}$, in those clusters depend primarily on the distance, $R_{n}$, from the center of the cluster to the shell $n$ in which they are located, not on the orientation of the atoms in shell $n$ with respect to the central $\mathrm{Ni}$ subcluster. The values of those moments are shown in Figs. 2 and 3 as a

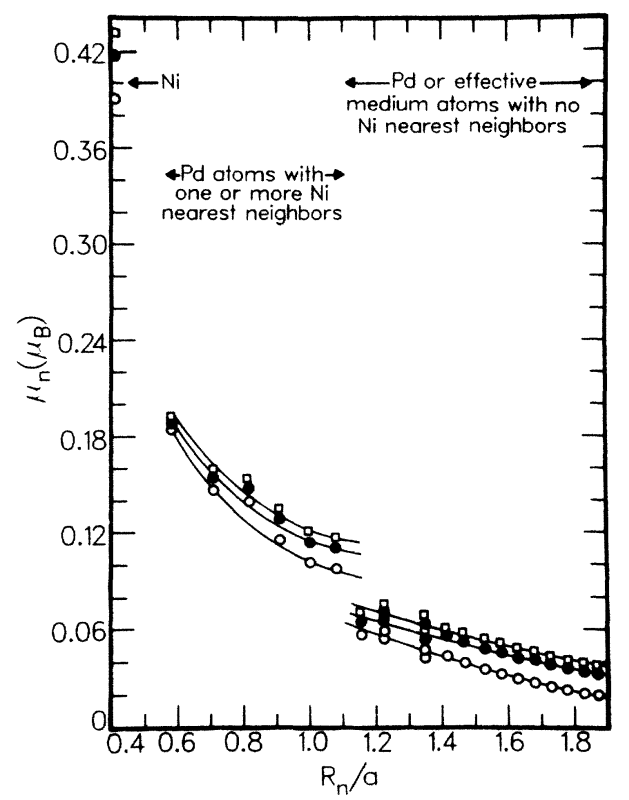

FIG. 2. Distribution of the zero-temperature spontaneous local moments, $\mu_{n}$, as a function of the ratio of distance away from the cluster center to the Pd lattice constant for a cluster containing a nucleus of three nearest-neighbor $\mathrm{Ni}$ impurities surrounded by $44 \mathrm{Pd}$ atoms and embedded in pure $\mathrm{Pd}$ (open circles) and effective media with $\bar{\chi}(0) / \tilde{\chi}(0)$ equal to 4 (closed circles) and 6 (open squares), respectively.

function of the ratio of $R_{n}$ to $a$, the $\mathrm{Pd}$ lattice constant, for the first and third clusters embedded in pure $\mathrm{Pd}$ and in effective media with uniform static susceptibilities, $\bar{\chi}(0), 4$ and 6 times that of pure Pd, $\tilde{\chi}(0)$.

Figure 4 shows the variation of the total giant moment $\mu$ associated with the first cluster as a function of $\tilde{\chi}(0) / \widetilde{\chi}(0)$ for the cases in which the giant polarization cloud associated with the cluster (i) is effectively cut off

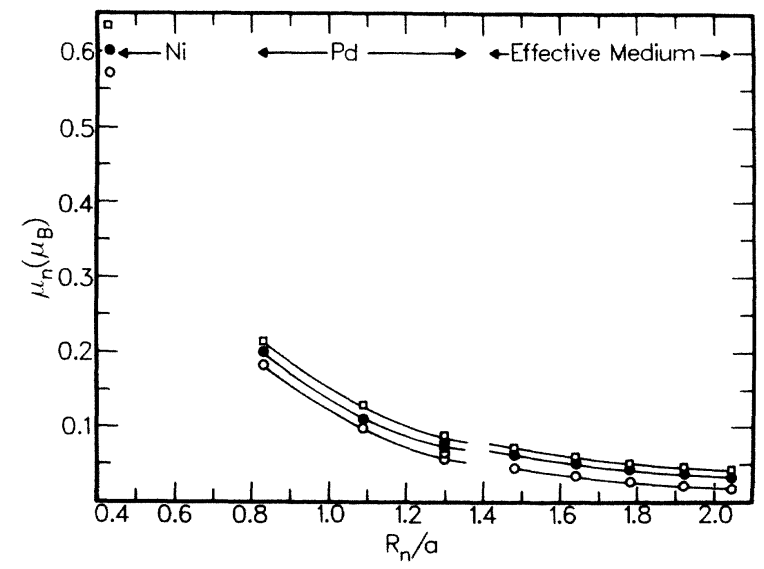

FIG. 3. Distribution of the zero-temperature spontaneous local moments, $\mu_{n}$, as a function of the ratio of distance away from the cluster center to the Pd lattice constant for a cluster containing a nucleus of four nearest-neighbor $\mathrm{Ni}$ impurities surrounded by $40 \mathrm{Pd}$ atoms and embedded in pure $\mathrm{Pd}$ (open circles) and effective media with $\bar{\chi}(0) / \tilde{\chi}(0)$ equal to 4 (closed circles) and 6 (open squares), respectively. 


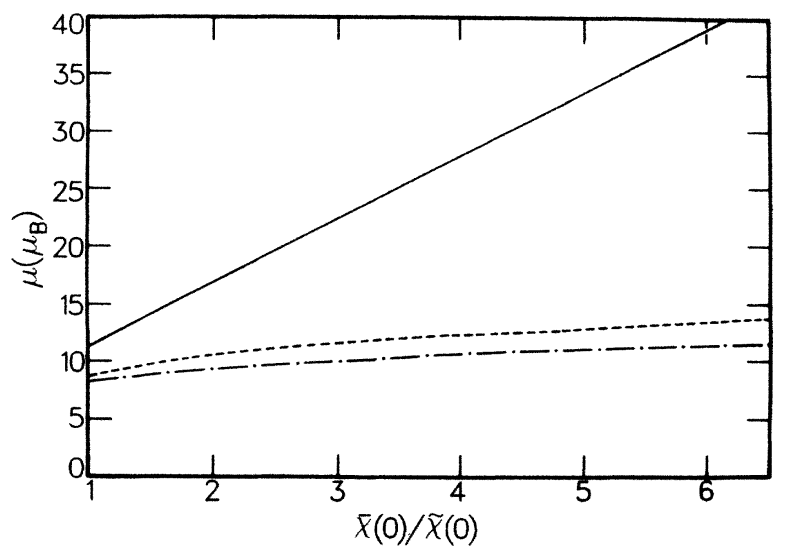

FIG. 4. Giant cluster moment nucleated by a nearestneighbor Ni triplet plotted vs the ratio of the uniform susceptibility of the effective medium in which the cluster is embedded to that of pure $\mathrm{Pd}$. The giant polarization cloud is allowed to extend out to infinity (solid line), and to distances of $9.95 \AA$ (280 sites) (dashed line), and $9.0 \AA$ (210 sites) (dash-dotted line), respectively.

at distances $R_{N}=9.0 \AA$ ( $N=210$ sites) or $R_{N}=9.95 \AA$ $(N=280$ sites) by Ruderman-Kittel-Kasuya-Yosida (RKKY) oscillations in $\bar{\chi}(\overrightarrow{\mathbf{r}})$, or (ii) is allowed to extend to infinity. It is seen that cutting off the clouds at a finite range has a large effect on the calculated magnetization, but that the calculated magnetization depends only relatively weakly on $N$. Figure 5 shows the variation of the calculated giant moments for each of the three clusters as a function of $\bar{\chi}(0) / \tilde{\chi}(0)$ for the case in which the polarization clouds are assumed to contain only 210 sites, as was found from percolation theory to be the case at the critical concentration for ferromagnetism in $P d$-Ni. For values of $\bar{\chi}(0) / \tilde{\chi}(0)$ of the order of

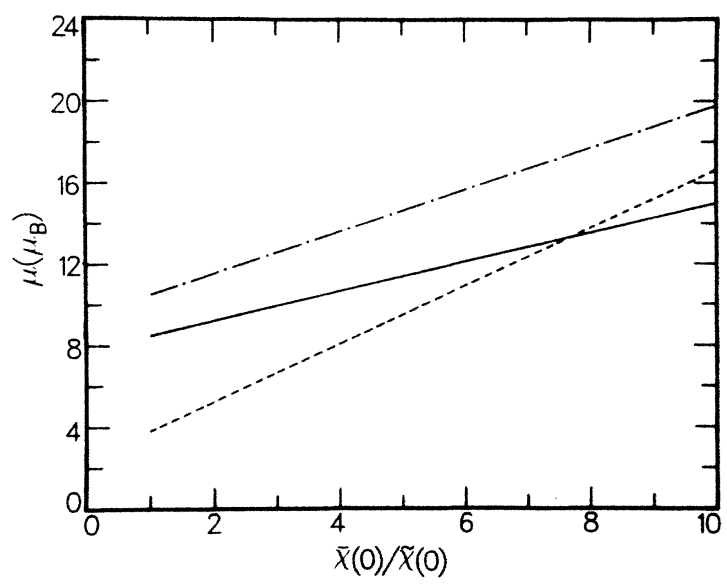

FIG. 5. The giant effective moment nucleated by a nearestneighbor Ni triplet (solid line), a linear chain Ni triplet (dashed line), and a nearest-neighbor $\mathrm{Ni}$ quartet (dashed-dotted line), plotted vs the ratio of the uniform susceptibility of the effective medium in which the clusters are embedded to that of pure Pd. For each of the clusters, the giant polarization cloud was allowed to extend only over a volume containing 210 sites.
$8-12$, which are appropriate for the range of $\mathrm{Ni}$ concentrations $(1.65<c<2.2$ at. \%) over which the value of $\mu$ is determined most accurately, the calculated values of $\mu$ are in good agreement with the experimental value ${ }^{15}$ of $17 \mu_{B}$.

The ratio $\chi_{n} / \tilde{\chi}(0)$ of the local susceptibility of atoms in the $n$th shell to that of atoms in pure Pd is shown in Fig. 6 as a function of the ratio $R_{n} / a$ of the radius of the $n$th shell to the Pd lattice constant for the third, and most magnetic, cluster embedded in pure $\mathbf{P d}$ and in effective media with $\bar{\chi}(0) / \widetilde{\chi}(0)$ equal to 2,4 , and 6 . Because the local band susceptibilities, $\Gamma_{i j}$, are much larger for $\mathrm{Ni}$ atoms than for Pd atoms and because the local moments on the Ni atoms are far from saturated, the local susceptibilities on the $\mathrm{Ni}$ atoms were found to be substantially larger than those on the close-in Pd atoms. The two different values shown at $R_{n} / a=1.3$ for $\bar{\chi}(0) / \tilde{\chi}(0)$ equal to 4 and 6 correspond to the $n=4$ and $n=5$ shells, which are inequivalent although they have the same radius. The slightly higher values of $\chi_{n}$, which are for the shell $n=4$, were determined nonlinearly, whereas $\chi_{5}$ was calculated within linear-response theory. The abrupt changes in $\chi_{n}$ found at the cluster boundary $\left(R_{n} / a \simeq 1.4\right)$ are approximately proportional to $\bar{\chi}(0) / \tilde{\chi}(0)$ and arise primarily from the change in the values of $u$ and/or the $\Gamma_{i j}$ as one passes from $P d$ to effective medium. Figure 7 compares the values of $\chi_{n} / \tilde{\chi}$ for the first cluster, with a nearest-neighbor $\mathrm{Ni}$ triplet, to those for the third cluster, with a nearest-neighbor $\mathrm{Ni}$ quartet, with both clusters embedded in an effective medium with $\bar{\chi}(0) / \tilde{\chi}(0)$ equal to 6 . The values of $\chi_{n} / \tilde{\chi}$ would be 1 and 6 , respectively, in pure $\mathrm{Pd}$ and in pure effective medium with $\bar{\chi}(0) / \tilde{\chi}(0)=6$. Note that those values are substantially affected both by partial magnetization of the atoms and by changes in their local envi-

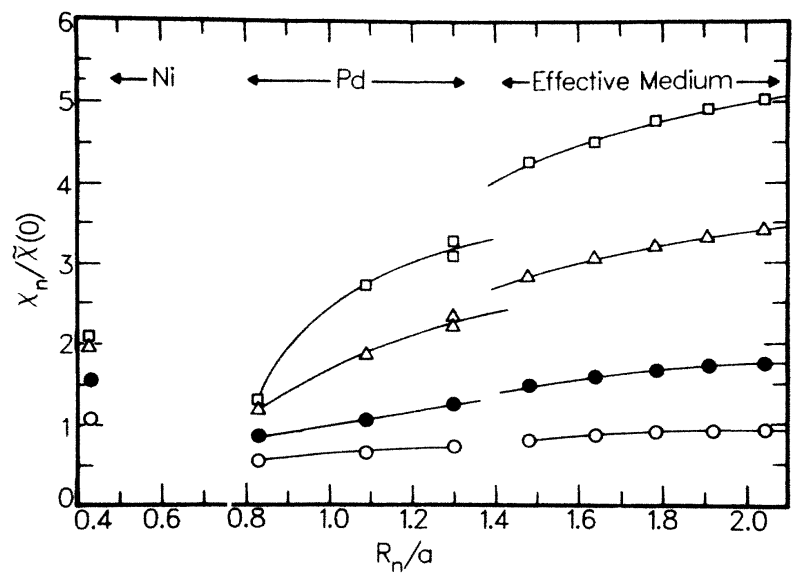

FIG. 6. Distribution of the zero-temperature exchangeenhanced local susceptibilities [normalized to $\tilde{\chi}(0)$ ] as a function of the ratio of distance away from the cluster center to the Pd lattice constant, for a cluster of four $\mathrm{Ni}$ impurities embedded in pure Pd (open circles), and in three effective media with $\bar{\chi}(0) / \tilde{\chi}(0)$ equal to 2 (closed circles), 4 (open triangles), and 6 (open squares), respectively. 


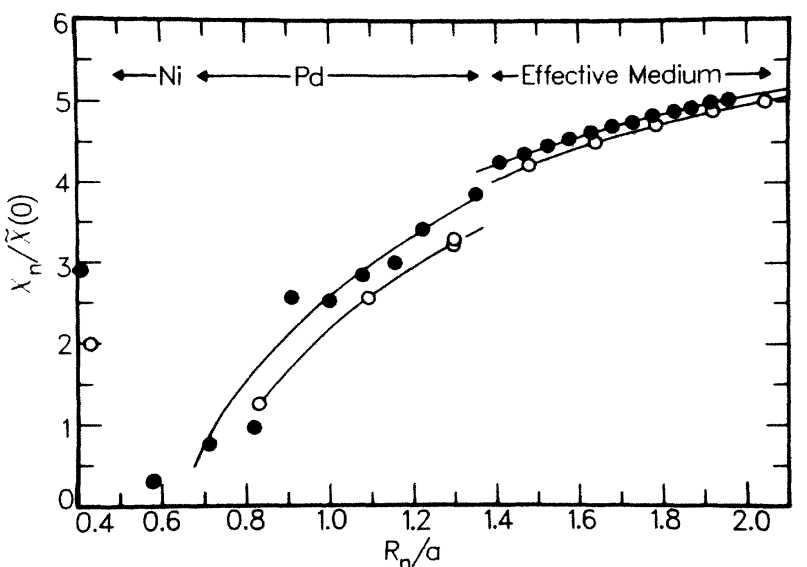

FIG. 7. Distribution of the zero-temperature exchangeenhanced local susceptibilities [normalized to $\tilde{\chi}(0)$ ] as a function of the ratio of distance away from the cluster center to the Pd lattice constant, for two different clusters, each embedded in an effective medium with $\bar{\chi}(0) / \tilde{\chi}(0)=6$. The first cluster contains a nearest-neighbor Ni triplet (closed circles) surrounded by $44 \mathrm{Pd}$ atoms; the second cluster contains a nearestneighbor $\mathrm{Ni}$ quartet (open circles) surrounded by $40 \mathrm{Pd}$ atoms.

ronment. Finally, Fig. 8 shows as a function of the uniform static susceptibility of an effective medium the fractional change, $\delta \chi(0 ; \mu) / \bar{\chi}(0)$, induced in that susceptibility by the embedding of each of the three clusters considered here.

\section{AN ANALYTIC INTERPOLATION SCHEME FOR $\mu$ AND $\delta \chi(0 ; \mu)$}

The numerical procedure for the determination of the giant cluster moment $\mu$ and the incremental susceptibili-

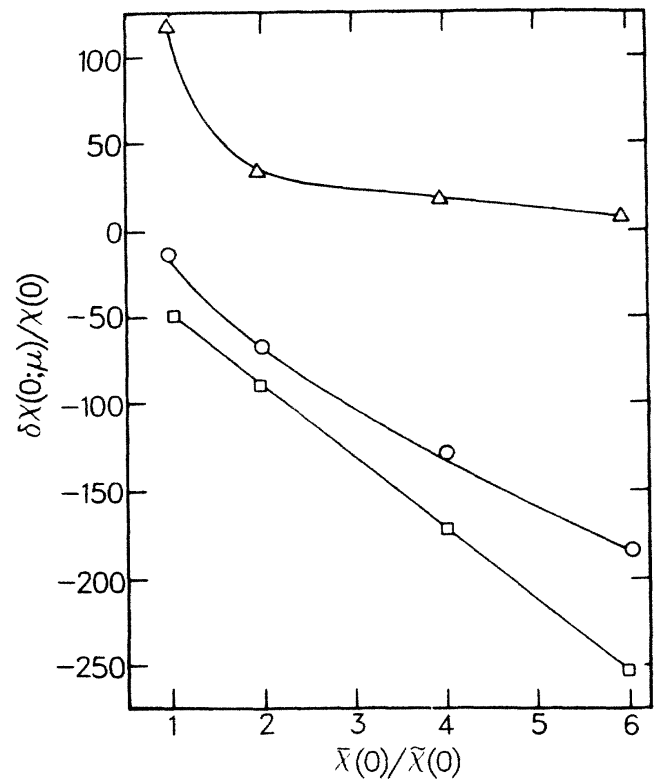

FIG. 8. The fractional change in the nonlinear exchangeenhanced uniform susceptibility per site introduced by embedding a nearest-neighbor Ni triplet (open circles), a linear chain $\mathrm{Ni}$ triplet (open triangles) and a nearest-neighbor $\mathrm{Ni}$ quartet (open squares) in an effective medium, plotted vs $\bar{\chi}(0) / \tilde{\chi}(0)$. ty $\delta \chi(0 ; \mu)$, which was presented and illustrated for three simple clusters in Sec. IV, in principle can be applied directly to a large representative sample of clusters with $\bar{\chi}(0)$ determined self-consistently so as to determine the susceptibility and magnetization of any alloy system. However, that would involve the direct calculation of $\mu$ and $\delta \chi(0 ; \mu)$ for hundreds or thousands of local configurations of atoms, most of which would involve clusters much larger and much less symmetrical than those considered in Sec. IV. Because of the highly nonlinear nature of the procedure developed in Sec. IV, that would be impractical in most cases. Therefore, we have developed simple interpolation schemes for the calculation of $\mu$ and $\delta \chi(0 ; \mu)$ for any cluster. These interpolation schemes give $\mu$ and $\delta \chi(0 ; \mu)$ as simple analytic functions of the uniform static susceptibility, $\bar{\chi}(0)$, of the effective medium in which the cluster is embedded and of $\delta \chi(0) / \bar{\chi}(0)$, the fractional change in $\bar{\chi}(0)$ induced by the cluster, as calculated within the linear RPA. Using the techniques of paper $I$, that requires only the inversion of a single $n \times n$ matrix for an $n$-site cluster, for either diagonal or off-diagonal disorder.

For either diagonal or off-diagonal disorder, the expressions for $\delta \chi(0) / \bar{\chi}(0)$ derived in I are inversely proportional to the determinant of the matrix $\mathbf{A}(0)$ to be inverted. For an embedded nonmagnetic cluster the determinant of $\mathbf{A}(0)$ is positive. For a sequence of increasingly more nearly magnetic clusters the inverse of the determinant decreases, vanishing at the critical point for moment formation and then becoming increasingly more negative for more and more strongly magnetic clusters. Thus, for a sequence of magnetic clusters, $|\delta \chi(0)|^{-1}$ must vanish at the point at which $\mu$ vanishes, and in general should be a smoothly varying monotonic increasing function of $\mu$. Also, by symmetry $|\delta \chi(0)|^{-1}$ must be an even function of $\mu$. In particular, $\mu^{2}$ must vanish linearly with $|\delta \chi(0)|^{-1}$, as for a uniform ferromagnet, and must approach a saturation value, $\mu_{s}^{2}$, for a fixed cluster size and fixed $\bar{\chi}(0)$, as $|\delta \chi(0)|^{-1}$ becomes large. Thus, $\mu$ should be given to a good approximation by an equation of the form

$$
\mu^{2}=\mu_{s}^{2} \tanh \left[M_{0}^{2} \bar{\chi}(0) / N|\delta \chi(0)| \mu_{s}^{2}\right],
$$

where the moment $M_{0}$ is a function of $\bar{\chi}(0)$. The factor of $N$, the total number of sites in the material, appears because $\delta \chi(0)$ is defined in such a way that it is proportional to $N^{-1}$. For clusters of more than 30 atoms, $\mu_{s}$ depends only weakly on cluster size for clusters containing a fixed number of $\mathrm{Ni}$ atoms, $n_{\mathrm{Ni}}$, and can be written in the form

$$
\mu_{s}=\mu_{s}^{(3)}+\left(n_{\mathrm{Ni}}-3\right)\left(\mu_{\mathrm{Ni}}^{\mathrm{sat}}-\mu_{\mathrm{Pd}}^{\mathrm{sat}}\right)
$$

where $\mu_{s}^{(3)}$ is a function of $\bar{\chi}(0)$ and where $\mu_{\mathrm{Ni}}^{\mathrm{sat}}-\mu_{\mathrm{Pd}}^{\mathrm{sat}} \simeq 0.64 \mu_{B}$.

Equations (5.1) and (5.2) were found to provide excellent fits to the values of $\mu$ calculated for the three clusters considered here with the parameters $\mu_{s}^{(3)}$ and $M_{0}$ given by the formulas

$$
\mu_{s}^{(3)}=\{12.5+13 \tanh [1.6 \bar{\chi}(0) / 13 \tilde{\chi}(0)]\} \mu_{B}
$$




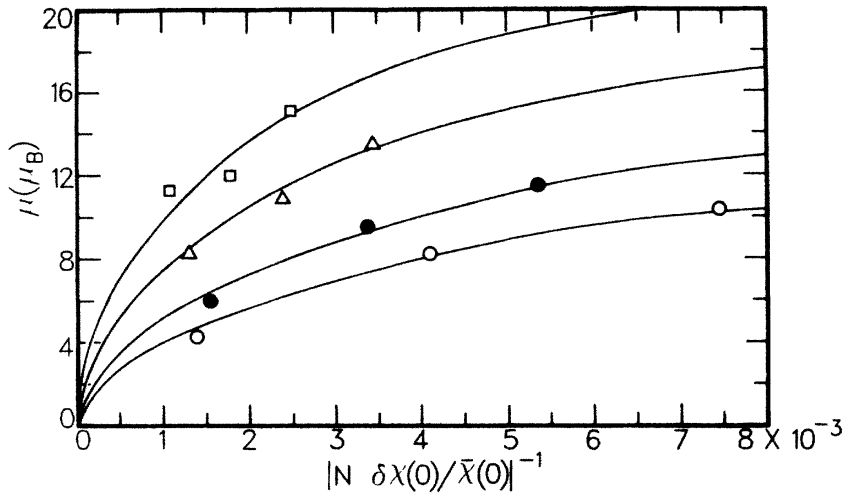

FIG. 9. The giant effective moment nucleated by the three $\mathrm{Ni}$ clusters embedded in pure $\mathrm{Pd}$ (open circles) and in three effective media with $\bar{\chi}(0) / \tilde{\chi}(0)$ equal to 2 (closed circles), 4 (open triangles), and 6 (open squares), respectively, plotted vs the inverse of the fractional change in the zero-temperature susceptibility associated with these clusters. The curves are the best fits of Eq. (5.1) to the values of $\mu$ for the three clusters.

and

$$
M_{0}=[88+37.5 \bar{\chi}(0) / \tilde{\chi}(0)] \mu_{B} .
$$

These fits are shown in Fig. 9 for $\bar{\chi}(0) / \tilde{\chi}(0)$ equal to 1 , 2 , 4, and 6. The values of $N \delta \chi(0) / \bar{\chi}(0)$ used in performing the fits were calculated using the values $\delta \mu \equiv u_{\mathrm{Ni}}-\tilde{u}=1.276 \mathrm{eV}$ for the case of diagonal disorder and $\bar{\delta}=0.536$ for the case of off-diagonal disorder within the geometric approximation; these values, which have been found to give a best fit to the experimental concentration-dependent Pauli susceptibility of $P d-\mathrm{Ni}$ alloys, gave essentially identical results.

We next consider the determination of the incremental susceptibility $\delta \chi(0 ; \mu)$ as an analytic function of $\delta \chi(0)$, $\bar{\chi}(0)$, and $n_{\mathrm{Ni}}$. For simplicity, we make the approximation of only off-diagonal disorder. ${ }^{17}$ For a homogeneous, translationally invariant material, within the RPA one can express the change, $\delta \chi(0)$, induced in the exchangeenhanced susceptibility by a change in the band susceptibility, $\Gamma(0)$, in the form

$$
\frac{\delta \chi(0 ; \mu)}{\chi(0 ; \mu)}=\frac{\delta \Gamma(0 ; \mu) / \Gamma(0 ; \mu)}{1-u \Gamma(0 ; \mu)[1+\delta \Gamma(0 ; \mu) / \Gamma(0 ; \mu)]} .
$$

By analogy with this expression we write the incremental susceptibility $\delta \chi(0 ; \mu)$, ensemble averaged over all clusters having the same size and the same giant moment, $\mu$, in the phenomenological form

$$
N \frac{\delta \chi(0 ; \mu)}{\bar{\chi}(0)}=\frac{N_{\mathrm{cl}} \delta \Gamma_{\mathrm{cl}}(0 ; \mu) / \bar{\Gamma}(0)}{1-u \bar{\Gamma}(0)\left[1+\delta \Gamma_{\mathrm{cl}}(0 ; \mu) / \bar{\Gamma}(0)\right]},
$$

where $N_{\mathrm{cl}}$ is the effective number of sites in the cluster and

$$
\delta \Gamma_{\mathrm{cl}}(0 ; \mu) \equiv \Gamma_{\mathrm{cl}}(0 ; \mu)-\widetilde{\Gamma}(0) .
$$

Here, $\mu$ is to be treated as an independent variable, not necessarily equal to the equilibrium moment on a cluster, and $N_{\mathrm{cl}}$ and $\delta \Gamma_{\mathrm{cl}}(0 ; \mu)$ are to be viewed as phenome- nological parameters.

In 'order to make Eq. (5.4b) useful, one must investigate the dependence of $N_{\mathrm{cl}}$ and $\delta \Gamma_{\mathrm{cl}}(0 ; \mu)$ on $\mu, \bar{\Gamma}(0)$, $\delta \chi(0), \bar{\chi}(0)$, and $n_{\mathrm{Ni}}$. The parameter $N_{\mathrm{cl}}$ should be of the order of, but somewhat smaller than, the sum of the number of atoms in the embedded cluster and the number of atoms within a correlation length of the embedded cluster. Fortunately, it turns out that $\delta \Gamma_{\mathrm{cl}}(0 ; \mu)$ depends on $N_{\mathrm{cl}}$ in such a way as to leave only a weak dependence of $\delta \chi(0 ; \mu)$ on $N_{\mathrm{cl}}$. The parameter $\delta \Gamma_{\mathrm{cl}}(0 ; \mu)$ must be an even function of $\mu$ by symmetry and thus can be expanded in the form

$$
\delta \Gamma_{\mathrm{cl}}(0 ; \mu)=\delta \Gamma_{\mathrm{cl}}(0)-\bar{\Gamma}(0)\left(b \mu^{2}+d \mu^{4}+e \mu^{6}+\cdots\right),
$$

where $\delta \Gamma_{\mathrm{cl}}(0), b, d, e, \ldots$ are parameters which in principle can depend on both $\bar{\Gamma}(0)$ and $\delta \Gamma_{\mathrm{cl}}(0)$. However, these parameters have been found to be essentially constant for fixed $\bar{\Gamma}(0)$, so that they may be regarded as functions only of $\bar{\Gamma}(0)$. Upon setting the cluster moment $\mu$ equal to zero in Eq. $(5.4 \mathrm{~b})$, we find that $\delta \Gamma_{\mathrm{cl}}(0)$ is given by the equation

$\delta \Gamma_{\mathrm{cl}}(0) / \bar{\Gamma}(0)=\frac{1-u \bar{\Gamma}(0)}{u \bar{\Gamma}(0)+N_{\mathrm{cl}}[N \delta \chi(0) / \bar{\chi}(0)]^{-1}}$

Upon substituting Eq. (5.6) into Eq. (5.5) and taking the limit of small moments so that one may neglect terms of order $\mu^{4}$ and higher in Eq. (5.5), one may use the familiar result

$$
\delta \chi(0 ; \mu) \sim-\frac{1}{2} \delta \chi(0)
$$

from the Landau theory of phase transitions and may use the asymptotic form,

$$
\mu^{2} \sim M_{0}^{2}|N \delta \chi(0) / \bar{\chi}(0)|^{-1},
$$

of Eq. (5.1), to obtain the result

$$
b=3 N_{\mathrm{cl}}[1-u \bar{\Gamma}(0)]\left[M_{0} u \bar{\Gamma}(0)\right]^{-2}
$$

Upon requiring $\Gamma_{\mathrm{cl}}(0 ; \mu)$ to vanish at the point at which $\mu$ reaches its saturation value, one obtains the further equation,

$$
\begin{aligned}
d \mu_{s}^{4}+e \mu_{s}^{6}+f & \mu_{s}^{8}+\cdots \\
& =\frac{N_{\mathrm{cl}}+N \delta \chi(0) / \bar{\chi}(0)}{N_{\mathrm{cl}} u \bar{\Gamma}(0)+N \delta \chi(0) / \bar{\chi}(0)}-b \mu_{s}^{2}
\end{aligned}
$$

which serves as a constraint on the coefficients of the terms of higher order in $\mu$. We found the series expansion $(5.5)$ for $\delta \Gamma_{\mathrm{cl}}(0 ; \mu)$ to converge rapidly, requiring at the most terms up through order $\mu^{8}$ to obtain good convergence. Thus, upon substituting Eqs. (5.6), (5.9), and (5.10) into Eq. (5.5) and then using Eq. (5.5) in Eq. (5.4b), one obtains a two-parameter analytic expression for $\delta \chi(0 ; \mu) / \bar{\chi}(0)$ for any magnetic cluster as a function only of $\bar{\Gamma}(0), \bar{\chi}(0), \delta \chi(0)$, which is easily calculated, and $N_{\mathrm{cl}}$, 


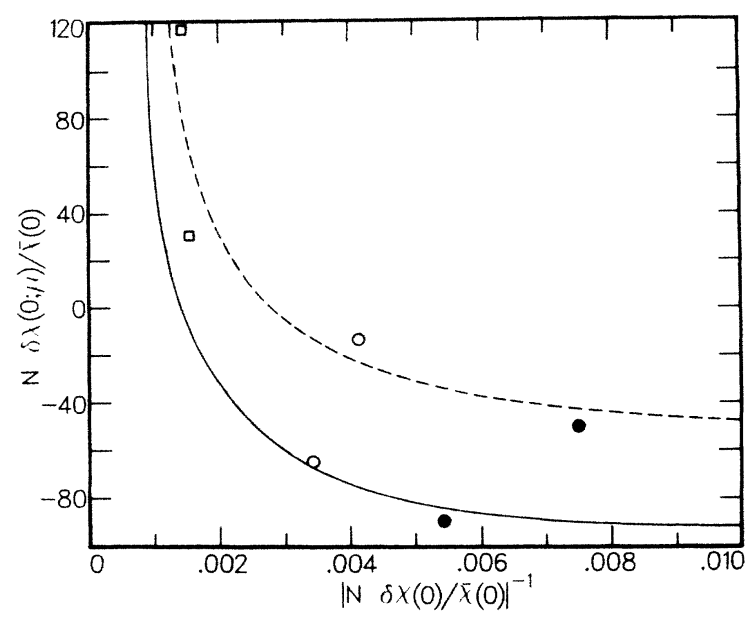

FIG. 10. The fractional change in the zero-temperature uniform nonlinear exchange-enhanced susceptibility as a function of the inverse RPA incremental susceptibility associated with magnetic Ni clusters embedded in pure $\mathrm{Pd}$ (dashed line) and in effective medium with $\bar{\chi}(0) / \tilde{\chi}(0)=2$ (solid line). The open squares, open circles, and closed circles refer to the values for the straight line triplet, compact triplet, and compact quartet, respectively.

which is known approximately. Furthermore, this expression is independent of $N_{\mathrm{cl}}$ to second order in $\mu$, because it must satisfy Eq. (5.7) of Landau theory to that order, and depends only weakly on $N_{\mathrm{cl}}$ even for moments as large as $0.8 \mu_{s}$. We found that the formula

$$
N_{\mathrm{cl}}=[5+13.3 \bar{\chi}(0) / \tilde{\chi}(0)] n_{\mathrm{Ni}},
$$

which is consistent with other estimates of $N_{\mathrm{cl}}$, such as that given by percolation theory at the critical concentration for ferromagnetism, allows a good fit to our calculated values of $\delta \chi(0 ; \mu)$ for all effective media for the three clusters for which full numerical calculations were performed.
Our numerical results for $\delta \chi(0 ; \mu) / \bar{\chi}(0)$ are shown in Fig. 10 as a function of $|\delta \chi(0) / \bar{\chi}(0)|^{-1}$ for $\bar{\chi}(0) / \tilde{\chi}(0)$ equal to 1 and 2 and for $n_{\mathrm{Ni}}=3$. Of course, from Eq. (5.7) $\chi(0 ; \mu)$ diverges as $\mu^{-2}$ as $\mu$ approaches zero, just as does $\chi(0)$. That is because the effects of spin fluctuations have not been included in this paper or in paper $I$. In the next paper in this series, we will show how the effect of spin fluctuations is easily incorporated in the present formalism.

\section{CONCLUSION}

We have generalized the formalism of the theory of the exchange-enhanced susceptibility of alloys so as to make it applicable to the nonlinear regime in which local moments are formed. We have then developed a technique based on that generalized formalism which allows the numerical calculation of (1) the total giant moment $\mu$ of any magnetic cluster embedded in an effective medium, and (2) the change $\delta \chi(0 ; \mu)$ in the susceptibility of the medium which is induced by embedding the cluster in it. We have illustrated the use of this technique by applying it to three different simple magnetic clusters of $\mathrm{Ni}$ and $\mathrm{Pd}$ atoms embedded in $\mathrm{Pd}$ and in different effective media. However, although this technique is much easier to use than would be the techniques of selfconsistent spin-polarized alloy band theory, it is still difficult and computationally time-consuming for the large clusters ( $\geq 30$ atoms) which must be considered in calculating the susceptibility and magnetization. Therefore, we have also developed parametrized, physically motivated simple analytic expressions for $\mu$ and $\delta \chi(0 ; \mu)$ for any cluster embedded in an effective medium. These analytic expressions allow one easily to calculate $\mu$ and $\delta \chi(0 ; \mu)$ for many thousands of local atomic configurations after determining the two parameters in each expression by fitting them to the results of full numerical calculations for a small number of atomic configurations.
*Permanent address: Department of Physics, California State University at Northridge, Northridge, CA 91330.

${ }^{1}$ N. Kioussis, J. W. Garland, and A. Gonis, preceding paper, Phys. Rev. B 37, 3611 (1988).

${ }^{2}$ R. Harris and M. J. Zuckermann, Phys. Rev. B 5, 101 (1972); T. Kato and M. Shimizu, J. Phys. Soc. Jpn. 33, 363 (1972).

${ }^{3}$ H. Hasegawa and J. Kanamori, J. Phys. Soc. Jpn. 31, 382 (1971); K. Levin, R. Bass, and K. H. Bennemann, Phys. Rev. B 6, 1865 (1972); J. Inoue and M. Shimizu, J. Phys. Soc. Jpn. 40, 1321 (1976); 42, 1547 (1977).

${ }^{4}$ D. J. Kim, Phys. Rev. B 1, 3725 (1970); H. Dvey-Aharon and M. Fibich, ibid. 10, 287 (1974).

${ }^{5}$ F. Brouers, F. Gautier, and J. van der Rest, J. Phys. F 5, 975 (1975); 5, 995 (1975); J. van der Rest, ibid. 7, 1051 (1977).

${ }^{6}$ P. Lederer and D. L. Mills, Solid State Commun. 5, 131 (1967); Phys. Rev. 165, 837 (1968); S. Engelsberg, W. F. Brinkman, and S. Doniach, Phys. Rev. Lett. 20, 1040 (1968).

${ }^{7}$ H. R. Krishnamurthy, K. G. Wilson, and J. W. Wilkins, Phys. Rev. Lett. 35, 1101 (1975); Phys. Rev. B 21, 1003 (1980).

${ }^{8}$ N. Kioussis, J. W. Garland, and J. S. Kouvel (unpublished).

${ }^{9}$ R. Podloucky, R. Zeller, and P. H. Dederichs, Phys. Rev. B
22, 5777 (1980); A. Oswald, R. Zeller and P. H. Dederichs, Phys. Rev. Lett. 56, 1419 (1986).

${ }^{10}$ B. Delley, D. E. Ellis, and A. J. Freeman, J. Magn. Magn. Mater. 30, 71 (1982); A. Rodriguez and J. Keller, J. Phys. F 11, 1423 (1981).

${ }^{11}$ Alloys such as $\mathrm{Ni}-\mathrm{Fe}$ of different ferromagnetic transition metals are of this type, provided that the majority-spin band is totally full.

${ }^{12}$ U. V. Barth and L. Hedin, J. Phys. C 5, 1629 (1972).

${ }^{13}$ G. G. Low and T. M. Holden, Proc. Phys. Soc. London 89, 119 (1966); T. J. Hicks, T. M. Holden, and G. G. Low, J. Phys. C 1, 528 (1968).

14J. W. Cable and H. R. Child, Phys. Rev. B 1, 3809 (1970).

${ }^{15}$ T. D. Cheung, J. S. Kouvel, and J. W. Garland, Phys. Rev. B 23, 1245 (1981).

${ }^{16}$ G. Chouteau, Physica 84B, 25 (1976).

${ }^{17}$ That is, we neglect the small difference between $u_{\mathrm{N}_{1}}$ and $u_{\mathrm{Pd}}$, keeping only the much larger differences between $\Gamma^{(\mathbf{P d})}(0)$ and $\Gamma^{(\mathrm{Ni})}(0)$ and, hence, between the local band susceptibilities $\Gamma_{i}^{(\mathrm{Pd})}$ and $\Gamma_{i}^{(\mathrm{Ni})}$. 